\title{
PARMENIDES: Facilitating Democratic Debate
}

\author{
Katie Atkinson, Trevor Bench-Capon, and Peter McBurney \\ Department of Computer Science \\ University of Liverpool \\ Liverpool, L69 3BX, UK \\ \{k.m.atkinson, tbc, p.j.mcburney\}@csc.liv.ac.uk
}

\begin{abstract}
This paper describes PARMENIDES, a system which facilitates structured debate about government policy.
\end{abstract}

\section{The PARMENIDES System}

The last two decades have seen a deliberative turn in the study of democracy in political philosophy [1]. Prior theories of democracy viewed ordinary citizens as no more than passive consumers of political information and argument, acting only when called upon to vote. In contrast, deliberative theories view citizens as producers of information, engaging as consenting and rational participants in reasoned argument with one another and with their political representatives. Thus, in this view, democracy is not simply a matter of periodic voting: it should also engage governments and the People in a process of continuous debate. Today, with the opportunities provided by the World Wide Web, communication is physically easier than ever before, but the long-standing problems that bedevil the effectiveness of communication remain. To be effective, communication must be clear, unambiguous and structured so that misunderstandings are minimised. In [2] we proposed a structure for persuasive argument that was intended to ease these communication problems, and to promote informed debate. In this paper, we describe a program which exploits this structure, and illustrate it with an example.

We start from an assumption that one party (say, the Government) has proposed an action or course of action, and presents a justification for this proposal to the other party, who may respond. The structure for the interaction between the two parties involves: a clear statement of the justification for an action, which makes explicit all the components of the reasoning underlying the argument; an opportunity to challenge any of the components and any of the inferential links between them; an opportunity for the proponent to respond to these challenges.

Within this dialogue structure, we see the justification for an action as involving the following argument scheme: an understanding of the current situation; a view of the situation which will result from performance of the action; features of the new situation which are considered desirable (the aspects which the action was performed in order to realise); the social goals which are promoted by these features (the reasons why they are desirable).

In [2], we advanced this structure for discussion and identified a number of ways in which it could be attacked. There, we identified fifteen distinct types of attack, several of 
Table 1. Table of Attacks

\begin{tabular}{|l|l|l|}
\hline Attack & Variants & Description \\
\hline 1 & 2 & Disagree with the description of the current situation \\
2 & 7 & Disagree with the consequences of the proposed action \\
3 & 6 & Disagree that the desired features are part of the consequences \\
4 & 4 & Disagree that these features promote the desired value \\
5 & 1 & Believe the consequences can be realized by some alternative action \\
6 & 1 & Believe the desired features can be realized through some alternative action \\
7 & 1 & Believe that an alternative action realizes the desired value \\
8 & 1 & Believe the action has undesirable side effects which demote the desired value \\
9 & 1 & Believe the action has undesirable side effects which demote some other value \\
10 & 2 & Agree that the action should be performed, but for different reasons \\
11 & 3 & Believe the action will preclude some more desirable action \\
12 & 1 & Believe the action is impossible \\
13 & 2 & Believe the circumstances or consequences as described are not possible \\
14 & 1 & Believe the desired features cannot be realized \\
15 & 1 & Disagree that the desired value is worth promoting \\
\hline
\end{tabular}

which had a number of variants according to the extent to which the attacker advanced a positive position in reply. Table 1 shows the attacks and the number of variants.

It is this variety of attacks which causes many of the problems in communication of views when using traditional means of correspondence. Our original intention was to implement a program controlling a computer mediated dialogue, in which the locutions would represent moves implementing the above attacks: this would ensure that each move was unambiguously identified with its intended effect. This program has been successfully implemented in JAVA, but evaluation has shown that, for casual users, many problems remain. Selecting the correct moves to realize a desired attack on a position is a task almost as difficult as correctly phrasing an attack in natural language. Essentially there is too much freedom of expression provided, and hence an overwhelming variety of options to select between. For this reason we have decided that if support is to be given to enable the general public to express their views as cogently as possible, some simpler form of interaction is required. These are exactly the problems encountered by earlier systems which have attempted to support democratic debate and dialogue. We address these usability problems by leading the user through a fixed series of moves; by constraining the choice of the user, the need for the user to understand the underlying model so as to make informed selection of moves is removed. Additionally, wherever possible statements are presented for approval or disapproval, reducing the problems associated with expressing the content of the various locutions. PARMENIDES is intended to realize these objectives.

The idea is to provide a simple web based interface which will guide the user in a structured fashion through a justification of an action giving opportunities to disagree at selected points. Each of these disagreements will represent one of the attacks above, so that the exact nature of the disagreement can be unambiguously identified. The users responses are written to a database so that information of what points of the argument are more strongly supported than others can be gathered. In the program described 
in this paper, PARMENIDES (Persuasive ARguMENt In DEmocracieS), we focus on negative criticism of the argument: in future work we will provide a similar interface to allow the construction of positive alternative arguments.

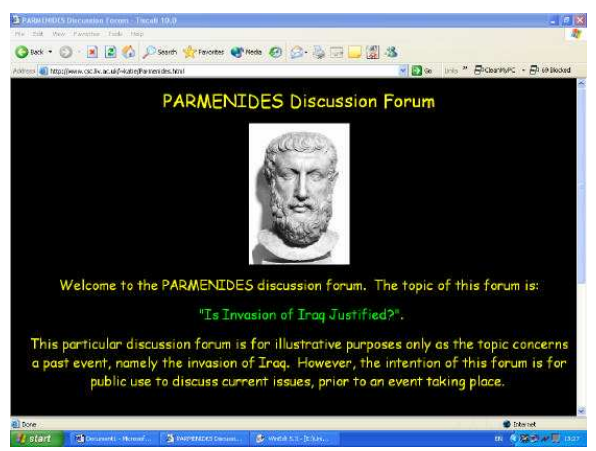

Figure 1: Introductory screen

PARMENIDES is implemented using PHP scripts and can be used at

http://www.csc.liv.ac.uk/ katie/Parmenides.html. The example debate concerns the invasion of Iraq in 2003. The aim of PARMENIDES is to present users with a position justifying a particular action and give them the opportunity to make a number of attacks on that position. We do not realize all of the attacks. Some of the attacks are directed against the soundness of the argument, and we here rely on the proponent of the position to produce only well formed arguments. Thus attacks 12, 13 and 14 are considered unnecessary, since we assume that the states of affairs and actions described are possible. Similarly we ignore attack 3 : whether the features are entailed by the consequences is a matter of logic, and we rely on the proponent to produce a sound position. Attacks 7, 8, 9 and 11 involve the proposal of some counter position: here we do not provide facilities to allow the statement of alternative positions, but concentrate on gathering a critique of the original position. Finally we ignore attack 10: this is a subtle matter and required in some domains, but since it does not vitiate the proposed action it is not required here. This leaves six attacks which we wish to allow. After an introductory screen, Figure 1, which takes some information about the user and provides some explanation about the purpose and use of the system, the user is presented with a structured statement of the position to be considered. At this point users can simply accept the argument in which case they are sent to a farewell screen. Otherwise, the user is then lead through a series of forms where they are given the opportunity to agree or disagree with the following elements, which comprise the initial position:

- the social values of the position (Attack 15),

- the promotion of the values by the desired consequences of the proposed action (Attack 4). Here they also have the opportunity to state consequences of the action which they believe compromises the desired value (attack 8),

- the consequences of the proposed action (Attack 2),

- the suggestion of alternative actions to realize the desired consequences (Attack 5).

- the description of the current situation (Attack 1).

- the user is then taken to the exit screen. 
The navigation above realizes six of the fifteen attacks possible against a position listed in Table 1. Each of these attacks proposes no positive information, and thus represents the simplest variant where several variants are possible. The six attacks represent a critique of the position proposed: if none of them can be made, then, provided the position is well formed, the position does represent a justification of the proposed action. Of the nine attacks not provided, four challenge the well formedness of the position (which we assume to be in order here), and, apart from the special case of attack 10, which does not dispute the action, the remaining attacks contest the action by developing a justification of an alternative action. We propose that these attacks are best provided by giving an opportunity to continue from the current exit screen, being prompted to extend the information already provided so as to develop a new position.

We are satisfied that PARMENIDES is usable by its target audience, and that it can effectively identify points of disagreement, and record them so that weight of opinion on various issues can be gauged. This is achieved without requiring the user of the system to have any particular familiarity with the underlying model of argument: the attacks are constructed from simple responses without any need for attacks to be explicitly formulated. Using PARMENIDES we can examine the acceptability of various parts of the position. For example, we are able to discriminate between those who support invasion for regime change from those who are concerned with international security. We can distinguish between those who believe that Saddam has no weapons of mass destruction from those who believe that he will disarm without invasion, from those who do not believe that he will use them. From this kind of information it is possible to see which elements of the argument need to be put more persuasively or better justified, and which elements could be emphasized to increase the acceptability of the argument.

The free text elements entered by the user are intended to be considered by a moderator who can consider whether they need to be added to the position. Thus if sufficient respondents see some particular circumstance as relevant it can be added to the list of circumstances displayed: if it is not believed by the moderator this is expressed by giving false as its default. We have envisaged use of PARMENIDES by the Government to justify its policy. A similar system could, however, be used by other bodies, such as pressure groups who could subject their own position to similar public scrutiny. The key advantage of PARMENIDES is that while it is firmly grounded on a model of argument, it does not require the user to understand and use that model; it therefore avoids the many usability problems encountered by previous systems with a similar ambition.

\section{Acknowledgments}

We are grateful to Sam Atkinson for his invaluable help in implementing PARMENIDES.

\section{References}

1. J. Bohman and W. Rehg, editors. Deliberative Democracy: Essays on Reason and Politics. MIT Press, Cambridge, MA, USA, 1997.

2. K. M. Greenwood, T. J. M. Bench-Capon, and P. McBurney. Structuring dialogue between the People and their representatives. In R. Traunmuller, editor, Electronic Government (EGOV 2003), LNCS 2739, pages 55-62, Berlin, 2003. Springer. 